\title{
LA VOZ AUTENTICA DE LOS NIÑOS Y NIÑAS EN LOS PROCESOS EDUCATIVOS FORMALES: UN DESAFÍO PARA LOS DOCENTES
}

The authentic voice of children in formal educational processes: a challenge for teachers

A voz autêntica de crianças em processos educacional formais: um desafio para os docentes

\section{Johanna Cortés Véliz}

Fundación Integra. Región de Coquimbo. Fono: +56 9 68449047. Correo electrónico: jcortes@integra.cl

\section{Resumen}

La infancia como categoría social y cultural, es concebida permanentemente como una etapa de preparación, valorándose por lo que llegará a ser y no por lo que es. Habitualmente es vista y escuchada a través parámetros que otorgan una mirada homogénea, bajo la premisa de una línea de progreso. A esta perspectiva le subyace una racionalidad técnica que comprende los procesos educativos formales desde categorías como el control, la eficacia, quedando supeditada a fines y resultados. Esta lógica impide a los docentes y adultos significativos escuchar de manera genuina la voz de los niños y niñas en sus múltiples lenguajes que aportan frescura y fertilidad a los proceso educativos.

La posibilidad de constituir una pedagogía que considere la voz auténtica de los párvulos ha de surgir de un pensamiento educativo que otorgue un reconocimiento de la legitimidad de la infancia en cuanto tal, en su existencia y forma de habitar el mundo, respetando y valorando el movimiento, la palabra, el gesto, para ello el docente ha de transitar por territorios en que la sutileza, la cautela y la atención le permitirán escuchar la voz de los niños y niñas, no para una apropiación instrumental de ésta, sino desde una postura hermenéutica para acoger y consecuentemente pensar una pedagogía desde una 
concepción práctica y ética que permitan al párvulo forjarse así mismo y desarrollar todo el potencial de aprendizaje que cada uno de ellos posee.

Palabras claves: Infancia, voz auténtica, escucha.

\begin{abstract}
Childhood as a social and cultural category is permanently conceived as a preparation stage, being valued by what it will become, but not by what it is. It is usually seen and heard through parameters that provide a homogeneous perspective, under the premise of a development line.
\end{abstract}

There is an underlying technical rationality to this perspective that understands formal educational processes from categories such as control, and efficiency, being subject to purposes and results. This logic avoids to teachers and other significant adults to genuinely listening to children and their multiple languages, that bring freshness and fertility to the educational processes.

The feasibility of building pedagogy that considers the authentic voice of children must come from an educational thought which recognizes childhood's legitimacy, as such, in their existence and way of inhabiting the world, respecting and valuing the word, face, gesture and movement. To do that, the teacher has to transit through territories where subtlety, caution and care will allow to hear the voice of children, not for its instrumental appropriation, but from a hermeneutic position to welcome it and consequently for thinking pedagogy from a practical and ethical conception that allows to every child forge to himself or herself and reaching their learning potential that each one possesses.

Key Words: Childhood, authentic voice, listening.

\title{
Resumo
}

Infầncia como categoria social e cultural é recorrentemente concebida como uma fase de preparação, sendo valorizada pelo que ela tornará-se, mas não pelo que ela é. Geralmente é vista e ouvida através de parâmetros que fornecem uma perspectiva homogênea, sob a premissa de uma linha de progresso. 
Há uma racionalidade técnica subjacente a esta perspectiva que compreende processos educativos formais desde categorias, tais como controle e eficiência, ficando sujeitos a fins e resultados. Esta lógica evita aos professores e outros adultos significativos a ouvir verdadeiramente às crianças e suas múltiplas línguas, que trazem frescura e fertilidade para os processos educativos.

A possibilidade de construir uma pedagogia que considera a autêntica voz das crianças deve vir de um pensamento educacional que reconhece a legitimidade da infância como tal, em sua existência e modo de habitar o mundo, respeitando e valorizando a palavra, rosto, gesto e movimento. Para isso, o docente deve trânsito por territórios onde sutileza, cautela e cuidado permitirá ouvir a voz das crianças, não para a sua apropriação instrumental, mas a partir de uma posição hermenêutica para acolher e, consequentemente, para pensar pedagogia desde uma concepção prática e ética que permite a todas as crianças se forjar eles mesmos e alcançar seu potencial de aprendizagem que cada um possui.

Palavras chaves: infância, voz autêntica, ouvir.

\section{La racionalidad dominante en las interacciones pedagógicas}

La infancia como categoría construida social y culturalmente ha transitado desde la concepción de niño como un adulto en miniatura a un sujeto de derechos; pasando por comprensiones que "atendieron al carácter psicobiológico, expresado en su madurez física e intelectual, que trajo consigo taxonomías, clasificaciones y determinismos socioculturales. Las herederas de esta implacable manera de observar a los niños y niñas fueron la psiquiatría, la psicología, la medicina y parcialmente la pedagogía" (Amador, 2012:75 en línea). El niño, niña, bebé como sujeto cognoscente es concebido en vías de su desarrollo, confiriéndose una mirada homogénea bajo la premisa de una línea de progreso fundamentada en leyes de carácter biológicas.

Esta concepción sigue estando representada en la idea de "la infancia como etapa de preparación, aprendizaje, dependencia y también de cierta irresponsabilidad, por lo que necesita ser protegida de los demás y también, en cierto modo, hasta de sí misma”(Gaitán, 2010:13). Esta perspectiva ha configurado creencias en los adultos que suponen que el niño necesita una estructura y requiere una dirección, se fundamenta en ello la idea que la 
educación ha de ser un proyecto fundacional, surgiendo la tentación de hacer del otro una obra propia, como señala Meirieu (1998) la educación aparece así como una fabricación. La tentación por querer acceder al misterio de la creación se hace presente, surgiendo entonces

la obstinación para que esta obra sea lo mejor posible, la cólera ante la resistencia del otro y la lentitud de sus progresos, el apasionamiento cuando las cosas empiezan a desbloquearse, $[\ldots]$ desaliento cuando se descubre que a fin de cuentas no se ha logrado nada, $[\ldots]$ serenidad al reemprender tranquilamente el trabajo y el éxtasis cuando el otro colma nuestros deseos (Meirieu, 1998: 33).

La racionalidad que sostiene estas creencias se fundamenta en una epistemología que se deriva de la filosofía positivista y defiende la idea que la práctica pedagógica se lleva a cabo mediante procesos de selección de los medios para determinados propósitos y mediante la aplicación de la teoría y la técnica que se derivan del conocimiento sistemático, preferiblemente científico (Schön, 1998).

Bajo este enfoque la práctica pedagógica en el aula se ve asociada a un intercambio como un proceso secuencial, llevado a cabo en espacios y tiempos determinados y controlada mediante procesos técnicos de planificación y evaluación (Calvo, 2007), sin considerar las referencias contextuales, pues el énfasis esta puesto en la búsqueda de estrategias didácticas, en el cómo de la enseñanza y no en el qué, ni para qué. La idea de práctica, en el sentido moderno del término, se plantea como una actividad productiva, un trabajo, que adquiere su valor por el efecto que se quiere conseguir, distinto del sujeto que actúa y está supeditada a fines, en la constante búsqueda de los medios mas apropiados para alcanzarlo (Bárcena, 2005).

Esto obedece a que la educación no se comprende como una posibilidad, sino como una realidad, pues las categorías de certeza y control, impiden comprender que "toda acción una vez iniciada, se escapa de las intenciones y la voluntad de su autor y entra en un juego de interacción y retroacción que puede modificar el curso de la acción” (Morín, 2016:43), pensar desde las categorías señaladas implica cerrarse a la posibilidad de la emergencia y la ocurrencia de los acontecimientos. 
El adulto como señala Maturana (1993) está centrado en una racionalidad que se orienta totalmente a la producción y la apropiación, encausándolo en un modo de vivir donde la atención se vuelve continuamente hacia los resultados de la acción, esta orientación trae consigo el continuo intento por controlar al otro y por tanto su negación.

La compulsión tan característica de nuestro tiempo de estar informados nos conduce a querer conocer el mundo antes de experimentarlo (Bárcena, 2005), la mayoría de los dispositivos que se han creado para proteger y resguardar la infancia, desde normas legales, planes curriculares, proyectos de intervención social, se conciben para un niño, cuya realidad ha de ser controlada, encauzada, disciplinada, moralizada, "el hombre moderno satura la experiencia, pero no porque se meta de lleno, sino porque la expulsa, la proyecta y la anula" (Fuentes, 2008: 62).

Una sociedad que inculca al niño cuanto antes una actitud razonable desde la lógica del adulto que es lineal y convergente, se convierte en un objeto de producción, ya que esto implica imponer un ritmo externo marcado por las tendencias e intereses de los adultos y no por el ritmo propio y natural del niño, esto es, sus necesidades, sus intereses distintivos de un período en el cual se busca conocer, comprender e imaginar el mundo, es un periodo de la vida en que el cerebro posee cualidades que lo hacen estar en mayor disposición de imaginar y aprender (Gopnik, 2010), un período donde es sensible al orden, al movimiento, al refinamiento sensorial, al lenguaje (Montessori, 1982), un período donde construye sus propias teorías (Gardner, 1996), sin embargo, el adulto sigue escuchándose a si mismo, proyectando en el niño sus propios intereses, esto es propio de una sociedad fría y calculadora como la que vivimos (L'Ecuyer, 2012), sin embargo y afortunadamente los niños y niñas se resisten como señala Meirieu (1998) se esconden o se rebelan, lo normal es que no se dejen llevar, simplemente para mostrarnos que los procesos educativos no son una fabricación, que no se es objeto en construcción sino un sujeto que se forja así mismo, por esto la pedagogía no consiste en que aquel que llega como una persona pueda ser moldeada al gusto de otro.

\section{El diálogo epistémico del niño con su entorno cultural y natural}


El bebé humano al nacer es amoroso en toda su estructura, toda su disposición anatómica y fisiológica está hecha para ser acogido, escuchado, para constituirse como humano primero en las coordinaciones sensoriales y motrices y luego en las coordinaciones consensuales como el lenguaje, por tanto en la medida que el bebé es escuchado a partir de su corporalidad, estableciendo diálogos sensoriales y motrices con el adulto significativo aprende a respetarse y respetar luego a otros, pues ha sido escuchado, reconocido y legitimado mediante el diálogo que surge en el juego en un emocionar coordinado entre el adulto y el niño (Maturana y Verden Zöller, 1993).

Al nacer el bebé es pura propensión aprender, no puede evitarlo y en la experiencia del vivir el desafío lúdico ocupa toda su capacidad, mientras lo hace no distingue entre interior y exterior (Calvo, 2007), implica un estado de atención permanente, es una actividad vivida en el presente en su realización y actuada emocionalmente sin ningún propósito exterior a ella (Maturana y Verden Zollër, 1993), si el niño no es interrumpido en su experiencia lúdica, es decir, es escuchado, visto y respetado en su hacer mantiene el entusiasmo como estado propio que moviliza su aprendizaje (Stern, 2013). Este estado de entusiasmo que manifiesta al aprender y que le permite sorprenderse, asombrarse y admirar, surge por el proceso de construcción de significados que hace del mundo que le rodea, esta es una de las razones por la cual los niños pueden formularse preguntas geniales, en ese sentido su vivir se asemeja al filósofo, pues mientras éste último deja suspendidas sus creencias y se abre a nuevas comprensiones, para él aun el mundo carece de significados (Flores, 2005).

Mirar la infancia por lo que es y no por lo que llegará a ser (Skliar, 2012) permite situarla más allá de los parámetros de la edad cronológica, sino como una forma de ser y estar en el mundo, esto implica comprender la epistemología del niño en primer lugar desde las categorías de unicidad, prescencialidad, encuentro, diálogo, incertidumbre, curiosidad y exploración, planteamiento, comprobación de hipótesis recurrente y emergencia de la palabra (Flores, 2005; López de Maturana, 2010). Y en segundo lugar desde la construcción de su identidad mediante, la simbolización permanente de su mundo a través del lenguaje, el juego, la representación bidimensional como el dibujo, la representación tridimensional como la modelación y la construcción, la expresión corporal y la música (Gardner, 1996) 
dichas expresión constituyen lo que Malaguzzi señala como los cien lenguajes del niño, cien manos, cien ideas, cien maneras de pensar, de jugar y de hablar ${ }^{1}$.

Actualmente existen variadas evidencias neurocientíficas que nos muestran que desde la primera infancia los bebés poseen grandes capacidades de adaptación al medio, creatividad, y afectividad, mas allá de las que se creía hace unas décadas (Gopnik, 2010). Las investigaciones muestran que los bebés tienen tendencia a razonar probalisticamente, (Bonatti, 2011) a probar hipótesis del mundo físico (Feigenson y Stahl, 2015) y tener una conducta empática frente a otros (Gopnik, 2010), lo que demuestra el gran potencial que los niños poseen y la predilección por lo inesperado, lo que constituiría una pulsión epistémica propia de la infancia que le permite entrar en diálogo a travès de la exploración del entorno. La afinidad del niño frente a los elementos naturales es una predisposición, asi lo sugiere la hipòtesis de la biofilia de Wilson (Cit. en Freire, 2015:19) que sostiene que el cerebro de los seres humanos esta programado para vincularse con otros seres vivos, por tanto posee una atracción congénita por la vida, un interés por la naturaleza en todas sus manifestaciones.

Los niños y niñas desde que nacen poseen la capacidad innata para comunicarse con sus cuidadores, saben escuchar y quieren ser escuchados por otros, son por esencia seres sociables, atraídos por los lenguajes de nuestra cultura, en la interacción con otros adultos cercanos, comienzan a descubrirse así mismos y al entorno, identificándose como personas singulares iniciándose en la expresión de su mundo interno: sus intereses, preferencias, gustos, emociones, sueños y fantasías mediante los diversos lenguajes que poseen.

\section{La presencia del otro y la escucha sensible}

La esencia de la educación como señala Arendt (cit. en Bárcena y Mélich, 2014) es la natalidad, el recién nacido es un recién llegado, alguien al que hay que iniciar, acompañar, acoger, esta relación se inscribe en una conexión cara a cara, que implica siempre la presencia del otro para un autentico actuar humano, esa interacción permite ver y ser vistos, percibir y ser percibidos, en el espacio de la pluralidad, esto es de igualdad y distinción.

\footnotetext{
${ }^{1}$ Los cien lenguajes de los niños es un poema que Loris Malaguzzi escribe para explicar su filosofía sobre múltiples lenguajes en los niños.

La voz autentica de los niños y niñas en los procesos educativos formales: un desafío para los docentes
} 
Este encuentro que se vive como una experiencia (Larrosa, 2006) implica abrirse a la posibilidad de hacer un viaje, en una actitud de reciprocidad, un movimiento hacia ese acontecimiento, que en definitiva ha de dejar una huella, una impronta y por tanto ha de ser significativo y trascendente, se trata de traer el mundo a la mano del niño, ofrecer una experiencia de aprendizaje mediado, esto es seleccionar del medio, interpretar y valorar los estímulos, facilitando en el individuo la creación de significados, nuevas relaciones y ampliando las posibilidades de comprensión del mundo. La mediación como señala Feuerstein (cit. en Prieto, 1989:30) se convierte así en un factor humanizador de transmisión cultural, cuyo punto de partida es un principio antropológico de creer en la potenciación y perfectibilidad humana.

La complejidad de la experiencia educativa esta dada por los múltiples factores sociales, contextuales e históricos que allí confluyen, así como por la emergencia de los sucesos, la fluidez de los acontecimientos y la subjetividad de los sujetos implicados, la educación desde esta perspectiva se concibe como acción y no como trabajo, desde la distinción que hace Arendt (cit. en Bárcena y Mèlich, 2014), este último tiene como fin la producción, es un proceso predictible de acuerdo al modelo, reversible y reproducible. Sin embargo, la educación supera esta práctica, pues se da en la pluralidad, imprevisibilidad, novedad radical, irreversibilidad, fragilidad y narración lo que permite al sujeto la formación de su identidad. Si entonces la educación es pensada como una acción humana en un espacio y un tiempo que no necesariamente son lineales y cronológicos, en interrelación e interacción con otros y cuyo fin es en si misma, la presencia del otro, ya sean adulto o par, es una condición primordial para ayudar a este recién llegado a forjarse así mismo, la ética del respeto por la vida que allí se crea y re crea permanentemente, ha de nutrir cada experiencia.

La dimensión comunicativa de la experiencia educativa, esta dada por el intercambio entre los participantes, no solo en la oralidad, sino en los múltiples lenguajes que se entrelazan: la corporalidad, el gesto, la mirada, la palabra, el ritmo, la tonicidad, el movimiento, el dibujo, la clave la tiene el educador que se convierte en oyente a partir de la escucha que hace del educando, en una disposición a interpretar las señales de los niños y niñas, "un educador ha de saber transitar espacios intermedios, espacios textuales [...] tiene 
que aprender el arte de descifrar las significaciones indirectas, el arte mismo de la hermenéutica" (Bárcena y Mèlich, 2014:108).

Por eso cuando el educador pregunta a los niños y niñas que quieren hacer, o que materiales les gusta, no logra aprehender la subjetividad, se mantiene en la literalidad sin lograr dilucidar los significados que tienen para ellos las múltiples experiencias en las que de manera diaria se involucra, los lenguajes de los niños requieren de una sutileza mayor que logre sensiblemente captar e interpretar los mensajes que se dejan entrever en las coordinaciones sensoriales, motrices y gestuales. Una escucha genuina se ve reflejada, por ejemplo en la observación atenta y solícita del educador que cuando los bebés golpean insistentemente la mesa con sus cucharas no acalla la experiencia con el silencio normativo, sino que comprende el interés y la necesidad de los niños por experimentar con el sonido y entonces ofrece una experiencia de aprendizaje mediado acercando el mundo sonoro a los niños y niñas.

La racionalidad que hoy prevalece en las educadoras no les permite estar atenta y receptiva a los mensajes frescos y genuinos que los niños y niñas expresan a partir de sus interacciones lúdicas y creativas, la apropiación que hacen de la voz de los niños, lejos de visibilizarlos los sitúan en una dimension instrumental.

Para escucharlos es necesario permitirse fluir de manera conjunta en un dominio de interacciones y emociones, en una intersubjetividad, que surge de existencias, posiciones epistémicas y vivencias diferentes. En este escenario el adulto ha de evitar el juicio anticipado, abriendose a la posibilidad de descubrir en el juego del niño, el complejo entramado de relaciones que establece de manera previa y durante la acción, que lo lleva y lo conduce por territorios que va experimentando y creando, este acercamiento no será factible de hacerlo desde parámetros homogéneos del desarrollo, tampoco desde la creencia de la incompletud, ni de la comparación con el adulto, pues siempre el niño en este caso estará en desventaja. El acercamiento ha de considerar que la niñez es una forma diferente de habitar el mundo, y por tanto esta interpretación de la actividad estará dada por la comprensión y valoración de lo propio de la niñez que es el asombro, la imaginacion, el aprendizaje, la incerteza. 
Cuando hay primacía del otro se puede cultivar la solicitud pedagógica que es una respuesta que implica estar actuando bajo la diferencia, demostrando una consideración afectuosa hacia el educando, se estima también como la capacidad para distinguir lo que es adecuado, implica una forma de reaccionar, escuchar y ver ante el niño (Van Manen, 2004), en la que prima el interés superior de éste, como fin último de las decisiones, significa apelar al buen juicio que se hace a partir de la reflexión en acción, en la que se ponderan las situaciones, factores y variables que allí confluyen, en un análisis que privilegia una capacidad para empatizar. "La subjetividad humana se constituye en la escucha y en la respuesta atenta a la voz del otro, una respuesta a su apelación y a su demanda" (Bárcena y Mèlich, 2014:151).

Un educador ha de observar y escuchar pedagógicamente, esto significa tener en cuenta la existencia total del niño, a partir de su contexto, su historia, su trayectoria vital, que le otorgan parámetros para el juicio y la decisión posterior, según sea el curso individual y singular de cada niño y niña.

\footnotetext{
La solicitud y el tacto son dos conceptos en estrecha relación. Alguien que habitualmente sea solícito es mas probable que demuestre ser una persona con un buen tacto en una determinada situación [...]parece que la solicitud pedagógica es una capacidad reflexiva, que nace de la reflexión detenida de las experiencias pasadas [...], pero el tacto en la enseñanza no es una simple destreza al contrario se puede definir como una preparación para la improvisación. [...] Es una actitud de la que poco se menciona en los textos y que sin embargo tiene que ver con la sensibilidad y sintonía. (Van Manen 2004: 49)
}

\section{La reflexión como posibilidad para la escucha atenta y sensible de los niños y niñas}

La educación de los niños y niñas significa una gran responsabilidad y merece por ello que los educadores sean profesionales que desarrollen un pensamiento reflexivo. En pedagogía necesariamente se requiere de la toma de decisiones, de la deliberación, lo que significa observar detenidamente las circunstancias educativas, las interacciones lúdicas para leer los contextos, las situaciones, problematizarlas y elaborar ideas, inferencias, sugerencias e hipótesis, poniendo a prueba las estimaciones o apreciaciones.

La experiencia reflexiva que se requiere para una pedagogía centrada en los niños y niñas que sea oportuna y pertinente, es eminentemente práctica, es decir, ha de estar 
orientada hacia las dimensiones valóricas del quehacer educativo, mas allá de lo normativo e instrumental, dado que este tipo de reflexión reside en explicar y clarificar las suposiciones y predisposiciones que subyacen en las situaciones prácticas y juzgar las consecuencias educativas que conlleva una determinada acción. Dado que en general los educadores vivencian situaciones que han de ser tratadas no solo desde el saber adquirido, que muchas veces no les sirve para enfrentar estas situaciones, las cuales requieren ser leídas y significadas. Por esto, es necesario repensar la racionalidad dominante desde una rememoración de la educación que permita volver a captar la experiencia como núcleo del hacer en educación (Bárcena, 2005).

Esta reflexión facilitará una actitud prudente, cautelosa y pertinente en la interacción pedagógica, ya que es posible preservar los sentidos profundos de la educación de párvulos que es acompañar y resguardar el desarrollo de niños y niñas en el plano físico, afectivo, espiritual, cognitivo, social, artístico, a partir de las características y necesidades propias de esta etapa, en un ambiente basado en el respeto y la legitimación del otro.

Es por tanto conveniente sostener la educación en una racionalidad de tipo práctica que se oriente a conjugar la acción sabia a favor de los niños y niñas, en un contexto valórico donde se privilegien los fines superiores.

Asumir una reflexividad práctica permitirá desarrollar propuestas pedagógicas pertinentes a las necesidades e intereses genuinos de los niños y niñas, con una visión ética centrada en la apreciación de la enseñanza no en función de los logros alcanzados, dado que la finalidad educativa no queda resuelta en objetivos expresados en resultados, sino en relación a los valores educativos, esto le otorga un carácter complejo a las situaciones pedagógicas, dado que los valores están sujetos a la reinterpretación permanente, lo que lleva a que la enseñanza se constituya en una aspiración la cual nunca es plenamente realizable, se convierte en una actividad que siempre es posible mejorar (Contreras, 1999).

Difiere bastante si la pedagogía se plantea sobre los intereses de los adultos al servicio de una racionalidad técnica o si se hace a partir de los intereses de los niños y niñas, para esto último los educadores requieren desarrollar una actitud solícita y un tacto en la enseñanza, como señala Van Manen (2004), lo que significa que los educadores han 
de transitar por territorios en la que la sutileza, la cautela y la atención le permitan escuchar la voz de niños y niñas, no para una apropiación instrumental de ésta, sino desde una postura hermenéutica acogiendo, respetando y legitimando las múltiples expresiones, para consecuentemente pensar la pedagogía desde una concepción práctica y ética que permita al niño o niña forjarse así mismo y desarrollar todo el potencial de aprendizaje que cada uno de ellos posee.

Una experiencia reflexiva es altamente favorable de realizarse en un espacio de encuentro profesional con los pares para compartir estas elaboraciones y someterlas a juicio, es allí, donde están las condiciones para abrir las conversaciones y para obtener puntos de vista diferentes, para argumentar, reconstruir y enriquecer las reflexiones personales, para avanzar en la comprensión de la subjetividad de los niños y niñas desde los acontecimientos que surgen de manera espontánea y original.

Estas nuevas distinciones de los dominios existentes en la realidad educativa, abrirán posibilidades de nuevas comprensiones a los adultos cerca de la ontología del niño, favoreciendo asi el desarrollo de un tacto pedagógico que le permita escuchar las señales que los niños y niñas dan permanentemente.

Cuando la práctica pedagógica se fundamenta en escuchar al educando se esta cultivando una tradición democrática ya que se ha de escuchar no solamente por amabilidad, si no por que existe el convencimiento que escuchar implica el gusto por un ejercicio democrático (Freire,1994).

La posibilidad de constituir una pedagogía que considere la voz auténtica de los niños y niñas ha de surgir de un pensamiento educativo que otorgue un reconocimiento de la legitimidad del niño, niña, en cuanto tal, en su existencia y forma de habitar el mundo, respetando y valorando el movimiento, la corporalidad, la palabra, el rostro, el gesto, y las múltiples formas de expresión, un pensamiento que no renuncia ante el tecnicismo que tiende a superar e invadir los procesos educativos, sino que abre posibilidades para la emergencia del otro con todo su potencial, novedad y singularidad, una pedagogía que posee un carácter ético por la preservación que allí ha de hacerse de la vida que se crea y se recrea permanentemente. 


\section{Referencias bibliográficas}

Amador, J. (2012). Condición infantil contemporánea: hacia una epistemología de las infancias. Pedagogía y Saberes, 37, 73-87. Consulta realizada 20 mayo 2016. http://revistas.pedagogica.edu.co/index.php/PYS/article/view/1882

Bárcena, F. Mèlich, J. (2015) La educación como acontecimiento ético. Natalidad, narración y hospitalidad. España: Miño y Dávila Editores.

Bárcena, F. (2005) La experiencia reflexiva en educación. Barcelona, España: Ediciones Paidós.

Bonatti, L., Téglá, E., Vul, E., Girotto V., Gonzalez, M., Joshua B. \& Tenenbaum, M. (2011) Pure Reasoning in 12-Month-Old Infants as Probabilistic Inference. Science 332, 1054-1059

Calvo, C. (2007) Del mapa escolar al territorio educativo. Disoñando la escuela desde la educación. La Serena, Chile: Ediciones Nueva Mirada.

Contreras, J. (1999) La autonomía del profesorado. Madrid, España: Ediciones Morata.

Fuentes, S. (2008) La filosofía de la infancia: acerca de la experiencia, el tiempo y el juego. Consulta realizada 3 julio 2016 http://reduei.com/wp-content/uploads/2012/06/CdCong.2008.pdf

Flores, P. (2004) El debate actual por la epistemología infantil o el ocaso de la ideología del pensamiento cognitivamente evolucionista. Consulta realizada 01 julio 2016 http://www.comie.org.mx/congreso/memoriaelectronica/v09/ponencias/at08/PRE11 78736866.pdf

Freire, H (2011) Educar en verde. Barcelona: Editorial Grao.

Freire, P. (1994) Cartas a quién pretende enseñar. Buenos Aires, Argentina: siglo XXI Editores.

Gardner, H. (1996) La mente no escolarizada. Cómo piensan los niños y como debiera enseñar la escuela. Buenos Aires: Editorial Paidós.

Gaitán, L (2010) Ser niño en el siglo XXI. Cuadernos de pedagogía, 407, 12-16 
Gopnik, A. (2010) El filosofo entre pañales: revelaciones sorprendentes sobre la mente de los niños y como se enfrentan a la vida. Barcelona: Editorial Temas de hoy

Larrosa, J. (2006) Sobre la experiencia. Aloma: revista de psicologia, ciències de l'educació i de l'esport Blanquerna 19, 87-112. Consulta realizada 01 julio 2016. http://www.raco.cat/index.php/Aloma/article/view/103367/154553

L’Ecuyer, C, (2012) Educar en el asombro. Barcelona: Editorial Plataforma.

López de Maturana, D. (2010) El juego como manifestación cuántica: una aproximación a la epistemología infantil POLIS, Revista Latinoamericana, 25, Consulta realizada 1 julio 2016. http://www.redalyc.org/articulo.oa?id=30512376014> ISSN 0717-6554

Maturana, H.; Verden Zöller G. (1993) Amor y juego, fundamentos de lo humano olvidado Ediciones Comunicaciones Noreste. Santiago, Chile.

Meirieu, P. (1998) Frankesteins Educador. Barcelona: Editorial Laertes.

Morin, E. (2016) Enseñar a vivir, manifiesto para cambiar la educación. Barcelona: Editorial Paidós.

Montessori, M. (1982) El niño, el secreto de la infancia. Editorial Diana: México

Prieto, M. (1989) La modificabilidad estructural cognitiva y el programa de enriquecimiento instrumental. Madrid: Bruño.

Schön, D. (1998) El profesional reflexivo. Como piensan los profesionales cuando actúa. Barcelona, España: Ediciones Paidós.

Skliar, C. (2012) La infancia, la niñez, las interrupciones. Revista Childhood \& Phylosophy 8 15, 67-81. Consulta realizada 16 julio 2016 http://www.periodicos.proped.pro.br/index.php/childhood/article/view/1244

Stahl, A. y Feigenson, L. (2015). Observing the unexpected enhances infants' learning and exploration. Science, 348, 91-94. Consulta realizada 27 junio 2016 http://aimeestahl.weebly.com/uploads/3/9/5/9/39595777/stahl_feigenson_science_2 015.pdf

Stern, A. (2013) Yo nunca fui a la escuela. España: Litera Libros Editorial 
Van Manen, M (2004) El tono en la enseñanza. El lenguaje de la pedagogía. Barcelona, España: Ediciones Paidós.

La voz autentica de los niños y niñas en los procesos educativos formales: un desafío para los docentes 